\title{
Harnessing wound healing and regeneration for tissue engineering
}

\author{
A.D. Metcalfe and M.W.J. Ferguson ${ }^{\mathbf{1}}$
}

UK Centre for Tissue Engineering (UKCTE), Faculty of Life Sciences, University of Manchester, Manchester, U.K.

\begin{abstract}
Biomedical science has made major advances in understanding how cells grow into functioning tissue and the signalling mechanisms used to achieve this are slowly being dissected. Tissue engineering is the application of that knowledge to the building or repairing of organs, including skin, the largest organ in the body. Generally, engineered tissue is a combination of living cells and a supporting matrix. Besides serving as burn coverings, engineered skin substitutes can help patients with diabetic foot ulcers. Today, most of these ulcers are treated with an approach that includes antibiotics, glucose control, special shoes and frequent cleaning and bandaging. The results of such treatments are often disappointing and ineffectual, and scarring remains a major problem, mechanically, cosmetically and psychologically. Within our group we are attempting to address this by investigating novel approaches to skin tissue engineering. We are identifying novel therapeutic manipulations to improve the degree of integration between a tissue engineered dermal construct and the host by both molecular manipulation of growth factors but also by understanding and harnessing mechanisms of regenerative biology. For the purpose of this summary, we will concentrate primarily on the latter of these two approaches in that we have identified a novel mouse mutant that completely and perfectly regenerates skin and cartilaginous components following ear injury. This experimental animal will allow us to characterize not only novel genes involved in the regeneration process but also to utilize cells from such animals in artificial skin equivalents to assess their behaviour compared with normal cells. This approach should allow us to create a tissue-engineered substitute, which more closely resembles the normal regional microanatomy and physiology of the skin, allowing better integration to the host with minimal or no scarring.
\end{abstract}

\section{Challenges of skin tissue engineering}

Tissue engineering can be defined as the development and manipulation of laboratory grown cells, tissues or organs to replace or restore the function of defective or injured body parts. Currently, there is a shortage of organs and tissues available for transplantation. There are many potential target organs for manipulation in tissue engineering, including not only the skin but the eye, liver, pancreas, bone and cartilage as well as vascular replacement grafts. Designing replacements to mimic the native tissue will improve the adequacy of tissue function, ultimately leading to improved patient care at less expense. The development of clinically useful tissues in vitro for application in a clinical situation is a complex task, determined by the nature of the tissues themselves. This complexity is also reflected in the interdisciplinarity of expertise required to produce such a tissue, in our case, skin. A platform of knowledge needs to be developed between cell and molecular biologists, biomaterials scientists as well as the clinicians and surgeons who will utilize the end-products. Not only do we need a biological understanding of the skin, equally important is the application of information from

Key words: MRL/MpJ, regeneration, repair, skin, tissue engineering, wound healing. Abbreviation used: TGF $\beta$, transforming growth factor $\beta$

${ }^{1}$ To whom correspondence should be addressed (email mark.w.ferguson@manchester. ac.uk). realistic engineering solutions to the clinical and surgical problems.

\section{Existing therapies and commercial products}

Cadaver skin is sometimes used as a temporary covering to protect the patient's recovering wounds but it is in limited supply, costly, and variable in quality with many safety concerns, e.g. viruses, transmissible spongiform encephalopathies etc. The alternative is to graft and mesh an autologous piece of skin from another unwounded location of the body. Neither of these approaches is ideal and has led to the development of alternative, off-the shelf commercial products.

The three main developments in tissue engineering of the skin are based on research data gained between 25 and 30 years ago. Rheinwald and Green [1] isolated and in vitro cultured keratinocytes from skin, Genzyme created living epithelial sheets using this technology, marketed since 1987 as EpiCel ${ }^{\circledR}$. Another development based on the study of Bell et al. [2], uses a collagen matrix seeded with dermal fibroblasts and covered with a layer of keratinocytes. This led to the development of substitutes like Apligraf ${ }^{\circledR}$, manufactured by Organogensis, and Dermagraft ${ }^{\circledR}$, created by Advanced Tissue Sciences. The final development was that of the acellular dermal substitute, Integra ${ }^{\circledR}$ by Integra Life Sciences based 
on the work of Burke et al. [3]. Tissue engineering has undoubtedly made great advances as a scientific discipline, it has however, been much less successful as a business. Three leaders in the industry fell into financial trouble very recently: Advanced Tissue Sciences, Organogenesis and Integra Life Sciences have all filed for bankruptcy. The reasons for these failures are multiple, one of the most cited was process automation costs. The costs of changing current medical practice were also identified as were the logistics of selling living products. However, there are major scientific drawbacks with existing products, which also need to be addressed. These include lack of vascularization or 'take', scarring, cell sourcing and persistence, absence of differentiated structures, biocompatibility and mechanotransductional properties, and ultimately the cost of both manufacture and supply.

\section{A clinical problem - simple repair or a regenerative medicine approach?}

In human and domestic animals scarring in the skin following trauma or surgery is a major medical problem. It often results in adverse aesthetics, loss of function, restriction of tissue movement and/or growth and adverse psychological effects [4]. At present, there are no prescription drugs for the prevention or treatment of dermal scarring. Within our group we are investigating novel approaches to tissue engineering in the skin, specifically to assist with the healing of wounds or replacement of tissue following burn injury. We are also identifying novel therapeutic manipulations to improve the degree of integration between a tissue engineered dermal construct and the host. This approach not only involves exploiting our prior expertise in the prevention of scarring but an understanding of regenerative biology and its application to a tissue engineering problem.

In higher vertebrates, injury to the skin initiates a cascade of events including inflammation, tissue formation and remodelling, which leads to a partial reconstruction of the damaged area. The wound repair process is initiated immediately following injury and the initial stages of inflammation involve polymorphonuclear leukocytes, mainly neutrophils, that are recruited to the wound site where their numbers increase steadily, peaking by $24-48 \mathrm{~h}$ [5]. Their main function appears to be phagocytosis of the bacteria that have been introduced into the wound during injury. The next cellular, immune element to be recruited to the wound are macrophages. They first appear within 48-96 h post-injury and generally reach a peak around the third day post-injury. During this time epithelial cells and keratinocytes migrate across the wound surface to restore epithelial integrity. T-lymphocytes then appear in significant numbers around the fifth day post-injury, with peak numbers occurring about the seventh day after injury. In contrast with polymorphonuclear leukocytes, the presence and activation of both macrophages and lymphocytes in the wound is critical to the progress of the normal healing process [5]. Macrophages release substances that facilitate the recruitment of additional inflammatory cells and aid the macrophage in tissue decontamination and debridement; in addition, growth factors and other substances are also released, which are necessary for the proliferation phase to occur. This involves the initiation and propagation of granulation tissue formation, which is composed of fibroblasts and inflammatory cells, along with new capillaries embedded in a loose extracellular matrix of collagen, fibronectin, hyaluronic acid and glycosaminoglycans. As the wound matures, collagen is initially deposited in a random arrangement that is subsequently reorganized, by cross-linking, into regularly aligned bundles oriented along the stress lines of the healing wound [5]. As remodelling progresses, there is a gradual reduction in the cellularity and vascularity of the reparative tissue, which results in the formation of a relatively avascular and acellular collagen scar.

There are, however, molecular differences between scarfree healing in embryonic wounds and scar-forming healing in adult wounds [4]. The inflammatory response in embryonic wounds consists of lower numbers of less differentiated inflammatory cells [6]. This together with high levels of morphogenetic molecules involved in skin growth and morphogenesis, means that the growth factor profile in a healing embryonic wound is very different from that in an adult wound [7-11]. Thus embryonic wounds that heal without a scar have low levels of TGF $\beta 1$ (transforming growth factor $\beta 1$ ) and $\beta 2$, low levels of platelet derived growth factor and high levels of TGF $\beta 3$ [4]. We have experimentally manipulated healing adult wounds in mice, rats and pigs to mimic the scar-free embryonic profile, e.g. neutralizing platelet-derived growth factor, neutralizing TGF $\beta 1$ and TGF $\beta 2$ or adding exogenous TGF $\beta 3[4,9,10,12-15]$. These experiments result in scar-free wound healing in the adult.

\section{Regeneration can occur in mammals}

Regeneration, unlike repair, leads to complete replacement of the injured structures, resulting in a fully functional, scarless, reconstructed tissue. Mammalian regeneration has long been considered the ultimate goal of the tissue engineering field. One of the challenges, however, is to understand and harness basic tissue biology, as well as tissue regeneration and the cellular and molecular mechanisms responsible for tissue turnover. Although numerous examples of complete regeneration exist in invertebrates, higher vertebrates such as amphibians demonstrate extensive but restricted regeneration, whereas mammals are severely limited in regenerative capacity.

To date, few examples of true mammalian regeneration have been described. The rabbit ear model extensively studied by Goss and Grimes [16] demonstrated a departure from the normal mammalian dermal repair process, with extended epithelial proliferation and migration, as well as dermal growth and the replacement of cartilage. More recently, Clark et al. [17] described similar ear punch regeneration in the inbred MRL/MpJ mouse strain. In this model, $2 \mathrm{~mm}$ excisional wounds in the ears of the MRL/MpJ mouse were found to regenerate completely after 4 to 5 weeks, whereas in the control strain, C57Bl/6, the wounds remained and did not 
Blastema-like structures either side of the punch biopsy hole. Thickened tip epithelia can be seen along with epidermal downgrowths (DG), cartilage (C), skeletal muscle and hair follicles (HF). Scale bar, $1 \mathrm{~mm}$.

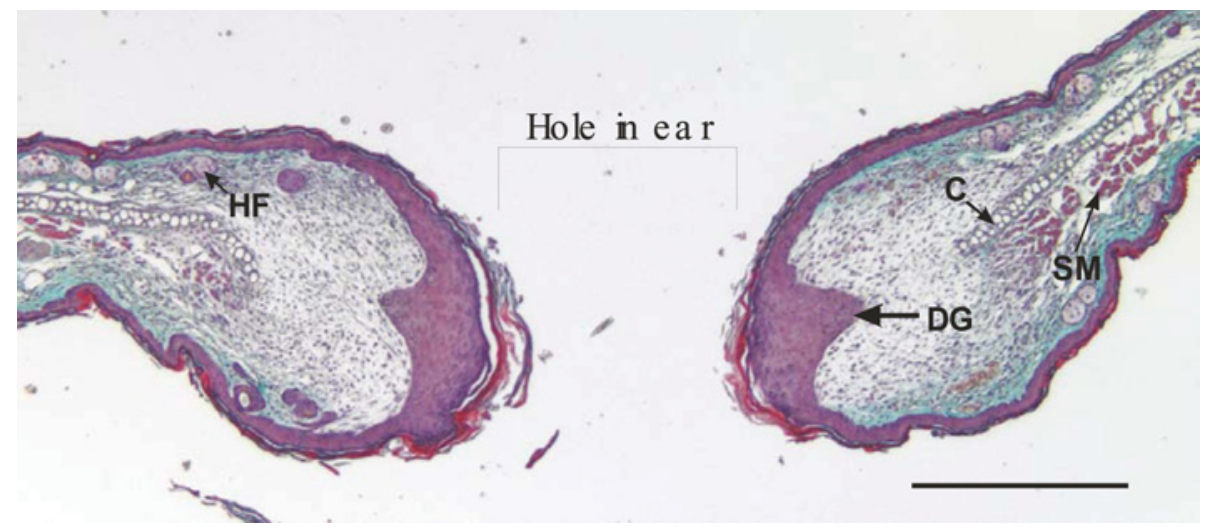

regenerate. The genetics behind this phenomenon have been studied, and genomic level QTL mapping has been performed [18]. This method demonstrated that candidate genes for ear regeneration were present on most of the mouse chromosomes, but failed to elucidate any true possible genes. Since that time, further studies have suggested that components of the inflammatory system may play a significant role in the regeneration/repair process [19-21].

We have demonstrated considerable variability in the MRL/MpJ regenerating mouse ear model [22]. The extent of complete regeneration was dependent on the degree of trauma imposed, with wounds created by a blunt ear punch least probable to regenerate compared with those created by a sharp surgical biopsy punch. Histological analysis suggests that the regenerative capacity of the MRL/MpJ mouse may only occur under specific conditions, and in situations where substantial amounts of necrosis and inflammation occurs, a default repair mechanism overrides the regenerative mechanism. It was also determined that the C57BL/6 control mouse did possess a limited regenerative capacity, with biopsy punch wounds closing to just over half their original size by the end of the time course. This was in contrast with the original phenotype description of these mice, where no significant decrease in wound size was observed in these animals. $\mathrm{MRL} / \mathrm{MpJ}$ mice regenerated their ears by developing a blastema-like structure, similar to the structures seen in regenerating amphibian limbs (Figure 1; [23-26]). Interestingly, the regeneration was characterized by development of de novo cartilage islands in the de-differentiated cells of the mesenchyme (Figure 2A). C57BL/6 wounded ears that did not regenerate, developed stunted bleb-like projections of cartilage contiguous with the wound site (Figure 2B; [22]).

\section{Regeneration and repair co-exist in the same animal}

We have recently demonstrated further variability in the $\mathrm{MRL} / \mathrm{MpJ}$ regenerative response. Using the same animals in which the ear punches were created, equivalent $4 \mathrm{~mm}$ dorsal
Figure 2 | MRL/MpJ and C57BL/ 6 ear wounds 35 days post-wounding

(A) Cartilage 'islands' clearly visible in the regenerating MRL/MpJ ear, and (B) cartilage wound edge forms stunted bulbous outgrowth in equivalently wounded C57BL/6 ears. Scale bar, $200 \mu \mathrm{m}$.
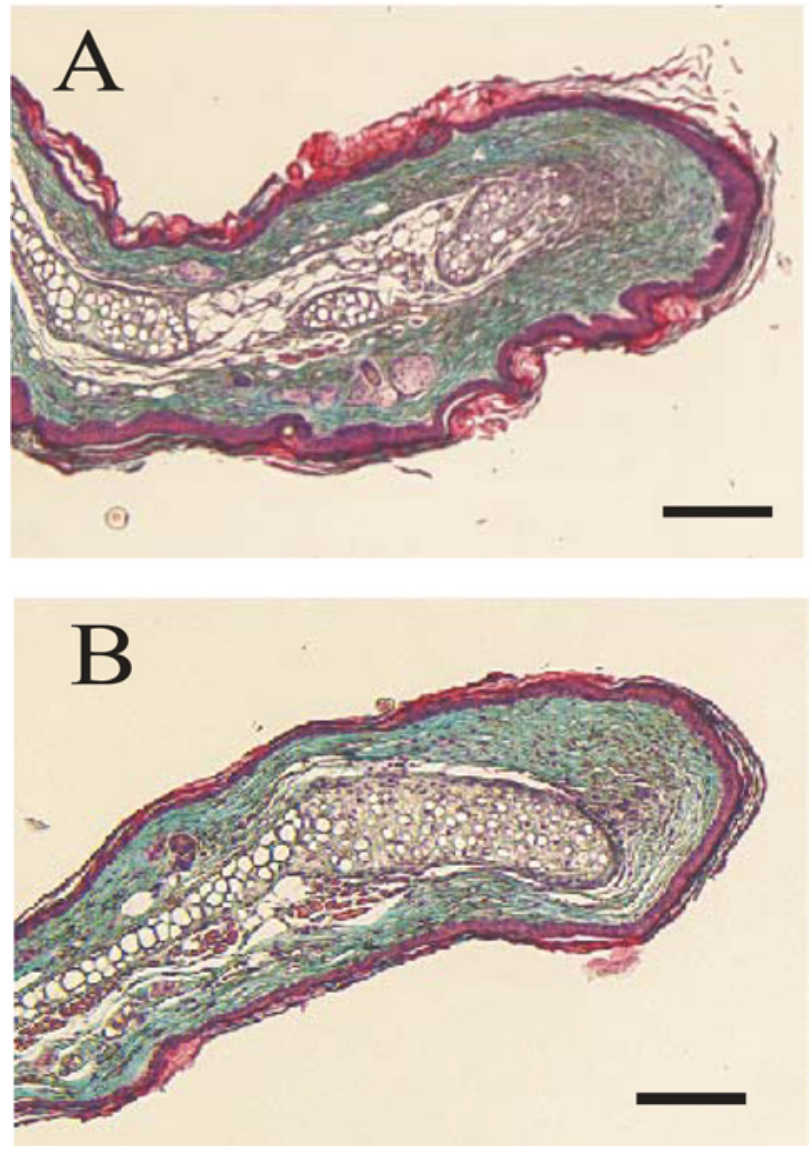

skin punch biopsy excisional wounds were also made. On analysis of those wounds on the dorsal skin of MRL/MpJ mice, no apparent regeneration occurred (Figure 3). Instead, 
Figure 3 | Ear and back wounds in MRL/MPJ mice 112 days postwounding

(A) Ear wounds showing epithelial fusion (arrow), and (B) back wound showing an absence of differentiated structures in the area of the wound. Scale bar, $500 \mu \mathrm{m}$.
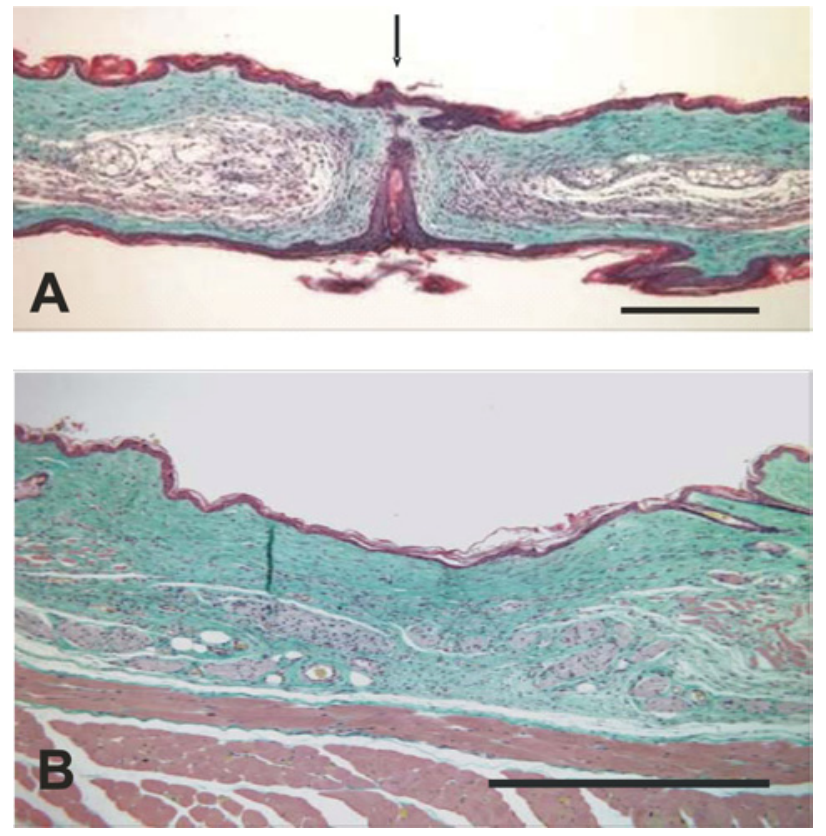

in the back wounds, scars of similar size and quality to those of the control animals (C57BL/6) developed. Analysis of early macrophage recruitment was found to be the same between the two strains as was the deposition of $\alpha$-smooth muscle actin. This suggests that the mechanism responsible for regeneration in the ear was not functioning in the skin.

This result was surprising since it contrasts quite markedly with earlier results observed in MRL/MpJ mice obtained after $2 \mathrm{~mm}$ through-and-through biopsy punching of the ear $[17,22]$. The regenerating blastema-like structures were not observed in our MRL/MpJ dorsal skin wounds but were seen in ear wounds created in the same animals [22]. Significantly, rather than regenerating like the ears, the dorsal wounds repaired with scarring. This demonstrates that repair in the back and regeneration in the ear of the same animal can occur (Figure 3). This fundamentally important phenomenon suggests that the mechanisms governing these two healing processes are probably controlled by similar molecules that diverge in different ways dependent on the location of injury [4].

Another process known to be used in response to injury and supposed to have a role in regeneration is apoptosis. Although little is known about apoptosis in the blastema, recently a study in planarians, has suggested that apoptosis may be involved in controlling cell numbers, eliminating unnecessary tissues or cells and remodelling the old tissues of regenerating body parts [27]. Bcl-2 family proteins are the main regulators of the apoptotic process, acting either to inhibit or promote it [28]. Pro- and anti-apoptotic Bcl-2 family members should function in harmony to regulate the apoptotic machinery, and their relative levels are critical for cell fate throughout development [29-31]. Further study is underway to understand the role of apoptosis in the repair and regeneration observed within the MRL/MpJ mouse.

This lack of regeneration in the dorsal skin is as yet undetermined, but may be a reflection of differences in the architecture of the two sites. The ear is a very thin structure with epidermis on both sides, as well as containing a supporting cartilage framework. The blastema-like structures that develop in the wounded ear are manifested adjacent to the cartilage at the wound edge. It may be that the regeneration of the ear is in some way controlled by a number of factors, including the deposition of new cartilage, glycosaminoglycans and extracellular matrix within the developing primitive mesenchyme of the blastema-like structure. In contrast, the skin on the body is loose, much thicker than the ear and with a substantial subcutaneous fat layer. Unlike the ear that has epithelia on both sides, there is only one layer of epithelia in the back. Contraction of the back wounds probably play an important role in the repair process, whereas in the ear this does not occur.

Mechanotransductive forces on cells are known to play a role in signalling pathways, growth factor shedding and cell proliferation $[32,33]$. It is supposed that cells are able to sense mechanical stress through autocrine loops localized to compliant extracellular spaces [32]. If this is the case, then in an injury made on the dorsal skin such cellular stresses are probably much different from the ones that impact on cells in the injured ear. This phenomenon may play a role in the subsequent mechanisms governing whether a wound closes by simple repair or by scar-less regeneration.

In summary, unlike the regeneration observed in the ears of MRL/MpJ mice, dorsal skin wounds heal with scarring similar to other strains of mice. The most exciting and significant finding of our present studies is that regeneration and repair can occur simultaneously within the same animal, as has also been noted to occur in humans [4]. This suggests that similar molecules and signals are probably required for both mechanisms but in some way are capable of being reprogrammed, dependent on the location of injury within the body.

\section{What will tissue engineers be capable of making in future?}

Similar to all new disciplines, expectation far outways the initial output, and the time to deliver a fully functional 'offthe-shelf' tissue engineered product is proving to be a lengthy process. More importantly, even before manufacturing and automation come into play, scientists need to clarify a number of critical issues. These include finding good sources of cells for the engineered tissues and organs, dealing with the immune system's response to them and their persistence, improving vascularization of the graft, building better matrix scaffolds, and learning how to better preserve the organs once they are constructed. Model experimental animals like the MRL/MpJ mouse demonstrate subtle differences between 
repair and regeneration and thus hold great hope for the tissue engineer because they are easily manipulated. Once fully understood, such model systems may yield an approach that will ultimately provide answers to some of the critical problems highlighted in the present study and raise hope for therapeutic regeneration in humans.

\section{References}

1 Rheinwald, J.G. and Green, H. (1975) Cell (Cambridge, Mass.) 6, 331-343

2 Bell, E., Ivarsson, B. and Merrill, C. (1979) Proc. Natl. Acad. Sci. U.S.A. 76, 1274-1278

3 Burke, J.F., Yannas, I.V., Quinby, Jr, W.C., Bondoc, C.C. and Jung, W.K. (1981) Ann. Surg. 194, 413-428

4 Ferguson, M.W. and O'Kane, S. (2004) Philos. Trans. R. Soc. Lond. B Biol. Sci. 359, 839-850

5 Chettibi, S. and Fergsuon, M.W.J. (1999) in Inflammation: Basic Principles and Clinical Correlates, 3rd edn (Gallin, J.I. and Snyderman, R., eds.), pp. 865-881, Lippincott Williams \& Wilkins, Philadelphia

6 Cowin, A.J., Brosnan, M.P., Holmes, T.M. and Ferguson, M.W.J. (1998) Dev. Dyn. 212, 385-393

7 Whitby, D.J. and Ferguson, M.W. (1991) Dev. Biol. 147, 207-215

8 Whitby, D.J. and Ferguson, M.W. (1991) Development 112, 651-668

9 O'Kane, S. and Ferguson, M.W.J. (1997) Int. J. Biochem. Cell Biol. 29, 63-78

10 Shah, M., Rorison, P. and Ferguson, M.W.J. (2000) in Scarless Wound Healing (Garg, H.G. and Longaker, M.T., eds.), pp. 213-226, Marcel Dekker, New York

11 Cowin, A.J., Holmes, T.M., Brosnan, P. and Ferguson, M.W.J. (2001) Eur. J. Dermatol. 11, 424-431

12 Shah, M., Foreman, D.M. and Ferguson, M.W.J. (1992) Lancet 339 213-214

13 Shah, M., Foreman, D.M. and Ferguson, M.W.J. (1994) J. Cell Sci. 107 1137-1157

14 Shah, M., Foreman, D.M. and Ferguson, M.W.J. (1995) J. Cell Sci. 108 985-1002
15 McCallion, R.L. and Ferguson, M.W.J. (1996) in The Molecular and Cellular Biology of Wound Repair, 2nd edn (Clarke, R.A.F., ed.), pp. 561-600, Plenum, New York

16 Goss, R.J. and Grimes, L.N. (1975) J. Morphol. 146, 533-542

17 Clark, L.D., Clark, R.K. and Heber-Katz, E. (1998) Clin. Immunol. Immunopathol. 88, 35-45

18 McBrearty, B.A., Clark, L.D., Zhang, X.M., Blankenhorn, E.P. and Heber-Katz, E. (1998) Proc. Natl. Acad. Sci. U.S.A. 95, 11792-11797

19 Li, X., Mohan, S., Gu, W., Miyakoshi, N. and Baylink, D.J. (2000) Biochim. Biophys. Acta 1524, 102-109

20 Li, F., Jin, F., Freitas, A., Szabo, P. and Weksler, M.E. (2001) Eur. J. Immunol. 31, 500-505

21 Li, X., Mohan, S., Gu, W. and Baylink, D.J. (2001) Mamm. Genome 12 52-59

22 Rajnoch, C., Ferguson, S., Metcalfe, A.D., Herrick, S.E., Willis, H.S. and Ferguson, M.W.J. (2003) Dev. Dyn. 226, 388-397

23 Echeverri, K., Clarke, J.D. and Tanaka, E.M. (2001) Dev. Biol. 236, 151-164

24 Rageh, M.A., Mendenhall, L., Moussad, E.E., Abbey, S.E., Mescher, A.L. and Tassava, R.A. (2002) J. Exp. Zool. 292, 255-266

25 Stocum, D.L. (2004) Curr. Top. Microbiol. Immunol. 280, 1-70

26 Endo, T., Bryant, S.V. and Gardiner, D.M. (2004) Dev. Biol. 270, 135-145

27 Hwang, J.S., Kobayashi, C., Agata, K., Ikeo, K. and Gojobori, T. (2004) Gene 333, 15-25

28 Metcalfe, A.D., Gilmore, A., Klinowska, T., Oliver, J., Valentijn, A.J. Brown, R., Ross, A., MacGregor, G., Hickman, J.A. and Streuli, C.H. (1999) J. Cell Sci. 112, 1771-1783

29 Metcalfe, A.D., Hunter, H.R., Bloor, D.J., Lieberman, B.A., Picton, H.M. Leese, H.J., Kimber, S.J. and Brison, D.R. (2004) Mol. Reprod. Dev. 68, 35-50

30 Sorenson, C.M. (2004) Biochim. Biophys. Acta 1644, 169-177

31 Cory, S., Huang, D.C. and Adams, J.M. (2003) Oncogene 22, 8590-8607

32 Tschumperlin, D.J., Dai, G., Maly, I.V., Kikuchi, T., Laiho, L.H., McVittie, A.K., Haley, K.J., Lilly, C.M., So, P.T., Lauffenburger, D.A. et al (2004) Nature (London) 429, 83-86

33 Yano, S., Komine, M., Fujimoto, M., Okochi, H. and Tamaki, K. (2004) J. Invest. Dermatol. 122, 783-790

Received 6 October 2004 